\title{
A STOCHASTIC SALVO MODEL FOR NAVAL SURFACE COMBAT ${ }^{1}$
}

\author{
Michael J Armstrong \\ Sprott School of Business, Carleton University, Ottawa, ON, K1S 5B6, Canada
}

\begin{abstract}
In this work we propose a stochastic version of the salvo model for modern naval surface combat. We derive expressions for the mean and variance of surviving force strengths and for the probabilities of the possible salvo outcomes in forms simple enough to be implemented in spreadsheet software. Numerical comparisons of the deterministic and stochastic models suggest that while the two models tend to provide similar estimates of the average number of ships surviving a salvo, this average by itself can be highly misleading with respect to the likely outcomes of the battle. Our results also suggest that a navy's preferences for risk (variability) and armament (offensive versus defensive) will depend on not only its mission objectives but also on whether it expects to fight from a position of strength or of weakness.

Subject classification: Warfare models: stochastic salvo combat. Tactics/strategy: naval surface tactics.

\section{INTRODUCTION}

A variety of analytical models of naval gunfire combat have been in existence for over a century in the form of Lanchester or Chase-Lanchester-Osipov (CLO) differential equation models (see Taylor 1983 for a review). However, since the middle of the 20th century warships have reduced their reliance on gunfire in favor of guided missiles such as the Exocets used against British warships in the 1982 Falklands campaign. To reflect

\footnotetext{
1 Published as: Armstrong MJ, 2005, "A stochastic salvo model for naval surface combat", Operations Research 53 \#5, 830-841, http://dx.doi.org/10.1287/opre.1040.0195
} 
this substantial change in military technology, Hughes (1995) proposed a new model of naval surface combat in which the primary exchange of fire was in the form of discrete salvos of missiles, rather than continuous streams of shells. His work illustrated the interaction between modern offensive and defensive firepower, and provided examples of how naval combat had in some sense become more lethal than before.

One of the strengths of this salvo model was its simplicity: the model's concepts and calculations were both straightforward enough to be understood and used by serving naval officers without extensive training in operations research. This transparency is important for military applications, as it allows the users to assess the logic of the model and ensure that it captures their view of the situation (Kent 2002).

On the other hand, a key limitation of the current salvo model is that its deterministic nature makes no provision for variation or uncertainty. This is a serious concern for a combat model, as most warfare is so chaotic as to be inherently stochastic; the user of a deterministic model therefore risks being mislead by its apparent predictability. As Ancker (1995, p 334) emphasized, "Any satisfactory model must, at the very least, yield a mean and variance of all pertinent outcome random variables. Anything less puts the decision maker (and the analyst) in peril of believing something which may have a high probability of being false." In the case of CLO gunfire models this motivation lead to the development of what is sometimes called the Markov Stochastic Lanchester model (see Kress \& Talmor 1999 for a recent example) as well as other stochastic extensions of CLO concepts. For the much newer salvo model there have been some numerical studies using simulation (Johns, Pilnick, \& Hughes, 2001; 
Lucas \& McGunnigle, 2003), but no analytical model has been produced to date for the stochastic case.

Our objective herein is to fill this gap by developing a salvo combat model in which random variation is incorporated into the model's main inputs and estimates are provided for the mean, variance, and probabilities of its key outputs. While so doing we also try to preserve as much as possible the ease of use and transparency of understanding of the deterministic salvo model; consequently our modeling decisions often reflect a preference for simplicity over detail. Note that our main focus is on battles involving moderately small to moderately large forces on each side. Small ship-versus-ship duels would lend themselves to a more specialized and detailed modeling treatment, while large theatre-level campaigns would be better served by a more strategic view.

Our work proceeds as follows. We begin in Section 2 by reviewing the deterministic salvo model of Hughes (1995) where we note that the offensive and defensive firepower of a task force are effectively summations of the corresponding individual ship characteristics. In Section 3 these per-ship firepower characteristics have their fixed parameters replaced by random variables; in a like manner the damage caused by each offensive missile also becomes a random variable. We then adapt some concepts from stochastic inventory models to derive the mean and variance of the surviving forces on each side as well as their probability of victory. Each of these measures is expressed in a form that can be implemented in user-friendly software such as a spreadsheet.

Section 4 uses numerical work to learn more about the model's behavior and implications. A comparison of the stochastic model's results with those of the original deterministic model suggests that both models provide similar estimates of the average 
surviving forces after a salvo. But because this average gives no indication of the variation in results the deterministic model can be quite misleading with respect to the potential number of survivors or the likelihood of victory. Thus the stochastic model provides additional insight that would be important to operational commanders planning their deployments, ship designers choosing the mix of weapon systems to install, and policy makers committing their citizen-sailors to an impending conflict.

Our work indicates that a navy's preferences for "risk" (variation) and for its mix of armament will depend upon its mission objectives and also on whether they expect to fight from a position of overall strength or of weakness. In general, a force that is superior in total firepower should try to balance its offensive and defensive capabilities, and then seek battle under conditions of greatest certainty (i.e. minimal variance) in order to "play the averages" that lie in its favor. Conversely, an inferior force should concentrate its resources on either offensive or defensive power (but not both) according to its mission objectives and then look for combat opportunities where the uncertainty is unusually high in the hope of getting a "lucky break".

We also find that the concept of combat lethality proposed by Armstrong (2004) is more complex when random variation is taken into account. In place of the three distinct categories of lethality that exist in the deterministic model we now find a continuous range of lethality levels with "strict" high lethality as a limiting extreme. Our discussion concludes in Section 5 with a summary of the model's contributions and limitations. The derivations of our results are presented in an Appendix.

\section{THE DETERMINISTIC SALVO MODEL}


In this section we briefly review the basic deterministic salvo model as defined by Hughes (1995); the reader is directed to that earlier article for a more thorough discussion of the model's terminology and underlying assumptions. Let parameter $A$ represent the force strength or number of ships on side A at the beginning of a battle. Each ship on side A has an offensive power rating $\alpha$, which is the number of well-aimed surface-tosurface missiles (SSMs) fired by each ship per salvo at the opposing force. Each of A's ships also has a staying power value $w$ that represents the number of SSM hits needed to achieve a firepower kill on it (i.e., to put it out of action for the remainder of the battle, though not necessarily sink it). Finally each ship has a defensive power value $y$, which is the number of incoming SSMs prevented from hitting per ship per salvo by active defenses such as surface-to-air missiles (SAMs). The ships on side B are similarly represented by parameters $B, \beta, x$, and $z$, respectively. Note that while this model is primarily intended for missile combat, it can also be used to model salvos of torpedoes, in which case the defensive power values will typically be zero.

The battle proceeds in discrete time intervals. In each interval side A fires a salvo from its offensive weapons at side B's ships while side B simultaneously fires a salvo of its own at side A. Each side uses its defensive fire to intercept the incoming offensive missiles, with any non-intercepted missiles causing damage to the targeted ships. The changes in force strengths from this exchange of salvos can be written as

$$
\Delta A=-(\beta B-y A) / w, \quad 0 \leq-\Delta A \leq A ; \quad \Delta B=-(\alpha A-z B) / x, \quad 0 \leq-\Delta B \leq B .
$$

An equivalent form for side $\mathrm{B}$ in terms of the surviving force strength $B_{1}$ is

$$
B_{1}=B-(\alpha A-z B)(1 / x), \quad 0 \leq B_{1} \leq B .
$$


From now on we describe the expressions only for side B since those for side A are structurally identical (exchanging parameters $\beta$ for $\alpha, y$ for $z$, etc.).

These equations highlight the interactive nature of offensive and defensive firepower. The number of hits suffered by side B is equal to the aggregated offensive power of side A minus the aggregated defensive power of side $\mathrm{B}$, if this difference is positive. The resulting loss of ships is determined by the number of hits sustained in conjunction with the amount of damage per hit. As an example, suppose that each side has $A=B=6$ ships with identical characteristics: $\alpha=\beta=4$ SSMs per salvo, $y=z=2$ SAMs per salvo, and $w=x=2$ hits allowed per ship (equivalent to $1 / 2$ ship lost per SSM hit). Equation (1) gives the number of surviving ships in this example as $A_{l}=B_{1}=6-[4(6)$ $2(6)](1 / 2)=0$, i.e. the first simultaneous exchange of missiles always results in the complete destruction of both forces.

\section{DEVELOPMENT OF A STOCHASTIC SALVO MODEL}

\subsection{Preliminaries}

Suppose that we have a set of independent and identically distributed (iid) random variables (rv) $K_{i}$, each of which has mean $\mu_{K}$ and variance $\sigma_{K}^{2}$, and that we wish to add $L$ of these together to get a new random variable $M$. Suppose further that $L$ is itself an integer random variable, independent of the $K_{i}$ and having mean $\mu_{L}$ and variance $\sigma_{L}{ }^{2}$. If all of these variables are non-negative then it is known (e.g. Ross 1993, pp 94 \& 98) that the mean and variance of the sum $M$ can be calculated as

$$
\mu_{M}=\mu_{L} \mu_{K} \text { and } \sigma_{M}^{2}=\mu_{L} \sigma_{K}^{2}+\sigma_{L}^{2} \mu_{K}^{2} \quad \text { where } M \equiv \sum_{i=1}^{L} K_{i}, \quad K_{i}, L \geq 0
$$


This relationship is often used (e.g. Chopra \& Meindl 2004, ch 11; Bagchi, Hayya, \& Chu, 1986) in stochastic inventory models when modeling the lead-time demand for a product. In that context, $L$ represents the number of days until the next shipment arrives while $K_{i}$ represents the demand on day $i$ for the product. It can be shown (Hadley \& Whitin 1963, p 153) that these same expressions hold when $L$ is a continuous variable, in which case the summation is replaced by an equivalent integral. For our purposes we shall need $L$ to be not only continuous but also possibly negative; consequently the expression for the mean of $M$ remains the same but a new one is needed for the variance.

LEMMA 1: VARIANCE OF A RANDOM SUM. Let L be a random variable with mean $\mu_{L}$, variance $\sigma_{L}^{2}$, probability density function (pdf) of $g(l)$, and cumulative distribution function (cdf) of $G(l)$. Also define $K_{i}$ to be non-negative iid $r v$ that are independent of $L$ with $E\left[K_{i}\right]=\mu_{K}$ and $\operatorname{Var}\left[K_{i}\right]=\sigma_{K}{ }^{2}$. Consider the random variable $M$ defined as a sum of $L$ of these $K_{i}$, i.e., if $L$ is integer we have

$$
M \equiv \sum_{i=1}^{L} K_{i} \text { if } L \geq 0, \quad M \equiv-\sum_{i=1}^{-L} K_{i} \text { if } L<0
$$

or more generally let $L$ be continuous with $E[K(l) \mid l]=l \mu_{K}$ and $\operatorname{Var}[K(l) \mid l]=|l| \sigma_{K}{ }^{2}$, so

$$
M \equiv \int_{0}^{L} K(l) d l \text { if } L \geq 0, \quad M \equiv-\int_{0}^{-L} K(l) d l \text { if } L<0
$$

so that in either case $M \geq 0$ if $L>0$ and $M \leq 0$ if $L<0$.

(a) The variance of $M$ can be calculated as

$$
\operatorname{Var}[M]=E[|L|] \sigma_{K}^{2}+\mu_{K}^{2} \sigma_{L}^{2}
$$


If $L$ is always non-negative then the expression simplifies to $\operatorname{Var}[M]=\mu_{L} \sigma_{K}^{2}+\sigma_{L}^{2} \mu_{K}^{2}$ as in Ross (1993). Conversely if L is always non-positive then the expression simplifies to $\operatorname{Var}[M]=-\mu_{L} \sigma_{K}^{2}+\sigma_{L}^{2} \mu_{K}^{2}$

(b) If L follows a normal distribution then an equivalent alternative expression is

$$
\operatorname{Var}[M]=\mu_{L} \sigma_{K}^{2}+{\sigma_{L}^{2}}^{2} \mu_{K}^{2}-2{\sigma_{K}}^{2} \mu_{L} G(0)+2{\sigma_{K}}^{2} \sigma_{L}^{2} g(0)
$$

\subsection{Model Development}

To develop the stochastic model we begin by rewriting Equation 1 for the deterministic model in an equivalent form in which each multiplication is replaced by a summation (if $A \& B$ are integer) or an integral (if $A \& B$ are allowed to be real).

$$
B_{1}=B-\sum_{k=1}^{N e t A B} v \quad \text { or } \quad B_{1}=B-\int_{0}^{N e t A B} v d t, \quad 0 \leq B_{1} \leq B
$$

where $v=1 / x$ is the number of ships lost due to each hit suffered, and

$$
\operatorname{Net}_{A B} \equiv O f f_{A}-\operatorname{Def}_{B} \quad O f f_{A} \equiv A \alpha=\sum_{i=1}^{A} \alpha=\int_{0}^{A} \alpha d t \quad \operatorname{Def}_{B} \equiv B z=\sum_{j=1}^{B} z=\int_{0}^{B} z d t
$$

Here $O f f_{A}$ represents the total offensive fire from side A, $D e f_{B}$ is the total defensive fire from side $\mathrm{B}$, and $\mathrm{Net}_{\mathrm{AB}}$ represents the offensive missiles from side $\mathrm{A}$ that are not intercepted by side B's defenses (see Table 1 for a summary of notation).

The next step is to redefine the number of well-targeted missiles successfully launched by each ship $i$ on side A to be a non-negative iid rv $\alpha_{i}$ with mean $\mu_{\alpha}$ and standard deviation $\sigma \alpha$. Here random variation may arise from imperfect reliability of the ship's physical systems (e.g. the firing button is pushed but the missile doesn't launch) or of its crew (e.g. when human beings make split-second decisions with limited information). Further variation with respect to the targeting of the missiles may be 
caused by the actions of the launching ship (e.g. the missile functions correctly but it is aimed in the wrong direction) or by those of the targeted ship (e.g. some form of long range electronic warfare (EW) distracts the missile off-course). Given this randomness on a per-ship basis, the offensive power for side A overall becomes a sum of random variables and thus will itself be random with mean and variance

$$
O f f_{A}=\sum_{i=1}^{A} \alpha_{i} \quad E\left[O f f_{A}\right]=\sum_{i=1}^{A} \mu_{\alpha}=A \mu_{\alpha} \quad \operatorname{Var}\left[O f f_{A}\right]=\sum_{i=1}^{A} \sigma_{\alpha}^{2}=A \sigma_{\alpha}^{2}
$$

where the summation is replaced by an equivalent integral if $A$ is non-integer.

As to the particular form of this randomness, it is natural to think of each ship as having a maximum number $n_{\alpha}$ of offensive missiles that it can fire simultaneously. This maximum may be prescribed by tactical doctrine or it may be due to a physical limit such as the number of missile launchers installed. If each round independently has a probability $p_{\alpha}$ of successfully being launched and well-targeted then the number of welltargeted rounds fired from each ship will follow a binomial distribution with mean $\mu_{\alpha}=$ $n_{\alpha} p_{\alpha}$ and variance $\sigma_{\alpha}^{2}=n_{\alpha} p_{\alpha}\left(1-p_{\alpha}\right)$. The number of well-targeted rounds $O f f_{A}$ for side A overall will then also have a binomial distribution with $\mathrm{E}\left[O f f_{A}\right]=A \mu_{\alpha}=A n_{\alpha} p_{\alpha}$ and $\operatorname{Var}\left[O f f_{A}\right]=A \sigma_{\alpha}^{2}=A n_{\alpha} p_{\alpha}\left(1-p_{\alpha}\right)$

For our purposes it will prove convenient to substitute a continuous normal distribution (with the same mean and variance) in place of the discrete binomial one. This is a well-established approximation whose accuracy can be improved through use of a continuity correction (Johnson \& Kotz 1969, pp 62-65); see the appendix for a comment on this issue. 
Next let the defensive power of each ship $j$ on side B be a non-negative iid $\mathrm{rv} z_{j}$. The effective defensive firepower on a given salvo may vary for many of the same reasons as offensive power (i.e., reliability, targeting, and EW) but also due to the inherent difficulty of successfully intercepting a rapidly moving target. A binomial distribution with firing limit $n_{z}$ and probability of successful intercept $p_{z}$ is a likely candidate for this variable and implies mean $\mu_{z}=n_{z} p_{z}$ and variance $\sigma_{z}^{2}=n_{z} p_{z}\left(1-p_{z}\right)$ for each ship. As with offensive power, we approximate the total defensive fire $\operatorname{Def}_{B}$ with a normal distribution having $\mathrm{E}\left[D e f_{A}\right]=B \mu_{z}=B n_{z} p_{z}$ and $\operatorname{Var}\left[D e f_{A}\right]=B \sigma_{z}^{2}=B n_{z} p_{z}\left(1-p_{z}\right)$.

The number of non-intercepted offensive missiles from side A that will hit side B is determined by subtraction: $\operatorname{Net}_{A B}=O f f_{A}-\operatorname{Def}_{B}$. We shall refer to $\operatorname{Net}_{A B}$ as the nominal number of missiles because this value can be negative if there is an excess of defensive power, whereas in such a case the actual number of remaining SSMs would be zero. Since $N_{A B}$ is the difference between two normal variables it follows a normal distribution itself, with mean and variance $\left(A \mu_{\alpha}-B \mu_{z}\right)$ and $\left(A \sigma_{\alpha}{ }^{2}+B \sigma_{z}{ }^{2}\right)$, respectively. As an illustration let us revisit the numerical example from the deterministic section. Choose $n_{\alpha}=8$ and $p_{\alpha}=0.5$ so that $\mu_{\alpha}=4$ SSMs per ship, and likewise choose $n_{z}=4$ and $p_{z}=0.5$ so that $\mu_{z}=2$ SAMs per ship. Then the nominal number of hits $\operatorname{Net}_{A B}$ will follow a normal distribution with $\mathrm{E}\left[\mathrm{Net}_{A B}\right]=24-12=12$ missiles and $\operatorname{Var}\left[\mathrm{Net}_{A B}\right]=12+6=18$ (or equivalently a standard deviation of 4.24 missiles).

The other random factor we wish to include is the damage caused by each nonintercepted offensive missile against its target. Hughes (2000, ch 6) describes some of the historical data on the variation seen in the damage per hit for various weaponry. On the one hand a single missile can sometimes be devastating: for example, the frigate $H M S$ 
Sheffield was sunk by a single SSM during the Falklands War (Eddy, Linklater, \& Gillman, 1982, pp 171-175). On the other hand an otherwise well-aimed missile might cause no damage whatsoever if it survives defensive fire but misses the target at the last moment due to e.g. an imperfect guidance system, evasive maneuvers by the target, or short range EW such as seduction chaff. To reflect this variation we let the loss (damage) suffered by side B due to missile $i$ be a non-negative iid $\operatorname{rv} v_{i}$ with mean $\mu_{v}$ and variance $\sigma_{v}{ }^{2}$. The default choice for the mean loss would naturally be $\mu_{v}=1 / x$ ships lost per hit inflicted, so that on average it would take a number of non-intercepted missiles equal to the ship's staying power to knock it out of action.

Combining the random number of nominal hits with the random amount of damage for each hit gives the total nominal loss suffered by side B from the salvo. This compound value is the sum of a random quantity $N e t_{A B}$ of random numbers $v_{i}$, so using Lemma 1 the mean and variance of this nominal loss can be calculated. We prefer instead to pass directly to the corresponding nominal surviving force strength $B_{1}$ *, obtained by subtracting the random nominal loss from the known starting strength $B$. PROPOSITION 1: NOMINAL SURVIVING FORCE STRENGTH. Let $g_{\text {NetAB }}(t)$ and $G_{N e t A B}(t)$ be the pdf and cdf respectively for the normal distribution describing the nominal number Net $t_{A B}$ of non-intercepted offensive rounds.

(a) The mean nominal surviving force strength $E\left[B_{1} *\right]$ will be

$$
\mu_{B 1 *}=B-E\left[N e t_{A B}\right] E[v]=B-\left(A \mu_{\alpha}-B \mu_{z}\right) \mu_{v} ;
$$

(b) The variance of the nominal surviving force strength $\operatorname{Var}\left[B_{1} *\right]$ will be

$$
\begin{gathered}
\sigma_{B 1^{*}}^{2}=\left(A \mu_{\alpha}-B \mu_{z}\right) \sigma_{v}^{2}+\left(A \sigma_{\alpha}^{2}+B \sigma_{z}^{2}\right) \mu_{v}^{2}-2 \sigma_{v}^{2}\left(A \mu_{\alpha}-B \mu_{z}\right) G_{N e t A B}(0) \\
+2 \sigma_{v}^{2}\left(A \sigma_{\alpha}^{2}+B \sigma_{z}^{2}\right) g_{N e t A B}(0)
\end{gathered}
$$


Note that Equation 3 for the mean mirrors the deterministic structure in Equation 1 in which fixed parameters have been replaced by averages. To see this in our ongoing numerical example set the average loss $\mu_{v}=1 / x=0.5$ ships per hit suffered as previously suggested and choose $\sigma_{v}=1 / 2.5 x=0.2$ ships. The nominal surviving forces on side B will average $\mu_{B 1 *}=6-(12 / 2)=0$ ships, which matches our earlier deterministic calculation, and will have standard deviation $\sigma_{B 1 *}=2.23$ ships.

At this point we again draw inspiration from basic stochastic inventory models where it is often assumed (e.g. Chopra \& Meindl 2004, p 340; Nahmias 1993, p 266) that if the length of the lead time and the demand per day are each normal and statistically independent, then the compound distribution for the total lead-time demand will also be normal. While not quite correct, this normality assumption is convenient because it makes inventory concepts and calculations both much easier to handle, especially for practitioners (e.g. logistics managers and business students), while still retaining the key concept of variation in both underlying components. This assumption has however been criticized (Bagchi, Hayya, \& Chu, 1986) as being unrealistic for some applications. One potential problem arises if the lead-time and demand per period are not statistically independent; for example, products that are temporarily in high demand at the retailer are often out of stock at the manufacturer and thus have a long lead-time precisely because of this high consumer demand. Another potential problem can occur if the lead-time distribution is discontinuous, as with a supplier who only delivers on a certain day each week. In the former case the actual shape of the compound distribution may be heavily skewed, while in the latter it will be multi-modal. 
We do not believe that either of these issues (statistical dependence or multimodality) is likely to be relevant in our context. Furthermore, with the salvo model already being a greatly simplified and aggregated model of combat we would argue that a simplifying assumption for the particular shape of a distribution is not only tolerable but actually preferable. Since we have already determined that the nominal number of hits is approximately normal, in the spirit of these basic stochastic inventory models we now assume that the compound distribution for the nominal loss on each side is also approximately normal, and so consequently is the nominal surviving force strength.

\subsection{Model Outputs}

Let us now consider how the nominal surviving force strength $B_{1} *$ can be used to derive the actual surviving force strength $B_{1}$. The actual figure is obviously limited to the range $[0, B]$ by the number of ships present, i.e., $B_{1}=\min \left\{\mathrm{B}, \max \left\{0, B_{1}^{*}\right\}\right\}$. When calculating the actual figure we also account for the fact that the number of missile hits is really an integer that is being approximated by a continuous distribution; thus we insert a continuity correction equal to one half the average damage caused by one missile, i.e. $\pm \mu_{v} / 2$. Alternative correction factors exist (Johnson \& Kotz, 1969, pp 62-65), but in the spirit of our modeling effort we choose the simplest one possible.

PROPOSITION 2: ACTUAL SURVIVING FORCE STRENGTH. Let $\mu_{B 1 *}, \sigma_{B 1}{ }^{2}, g_{B 1}(t)$, and $G_{B 1 *}(t)$ be the mean, variance, pdf and $c d f$, respectively, for the normal distribution that describes the nominal surviving force strength $B_{1}{ }^{*}$.

(a) The mean actual surviving force strength is

$$
E\left[B_{1}\right]=\int_{0+\mu_{v} / 2}^{B-\mu_{v} / 2} t g_{B 1^{*}}(t) d t+B\left(1-G_{B 1^{*}}\left(B-\mu_{v} / 2\right)\right)
$$


which can alternatively be expressed for calculation purposes as

$$
\begin{gathered}
=\mu_{B 1^{*}}\left[G_{B 1^{*}}\left(B-\mu_{v} / 2\right)-G_{B 1^{*}}\left(0+\mu_{v} / 2\right)\right]-\sigma_{B 1^{*}}{ }^{2}\left[g_{B 1^{*}}\left(B-\mu_{v} / 2\right)-g_{B 1^{*}}\left(0+\mu_{v} / 2\right)\right] \\
+B\left(1-G_{B 1^{*}}\left(B-\mu_{v} / 2\right)\right)
\end{gathered}
$$

(b) The variance of the actual surviving force strength is

$$
\operatorname{Var}\left[B_{1}\right]=\int_{0+\mu_{v} / 2}^{B-\mu_{\nu} / 2} t^{2} g_{B 1^{*}}(t) d t+B^{2}\left(1-G_{B 1^{*}}\left(B-\mu_{v} / 2\right)\right)-\left(E\left[B_{1}\right]\right)^{2}
$$

which can also be expressed as follows.

$$
\begin{gathered}
=\left(\mu_{B 1^{*}}^{2}+\sigma_{B 1^{*}}{ }^{2}\right)\left[G_{B 1^{*}}\left(B-\mu_{v} / 2\right)-G_{B 1^{*}}\left(0+\mu_{v} / 2\right)\right]+B^{2}\left(1-G_{B 1^{*}}\left(B-\mu_{v} / 2\right)\right)-(E[B])^{2} \\
-\sigma_{B 1^{*}}{ }^{2}\left[\left(B-\mu_{v} / 2+\mu_{B 1^{*}}\right) g_{B 1^{*}}\left(B-\mu_{v} / 2\right)-\left(0+\mu_{v} / 2+\mu_{B 1^{*}}\right) g_{B 1^{*}}\left(0+\mu_{v} / 2\right)\right]
\end{gathered}
$$

The reason for the alternative expressions in our results above and elsewhere is that they allow for calculations without the need for explicit integration. These equations can thus be implemented in e.g. Microsoft Excel spreadsheet software using the built-in function NORMDIST to evaluate both the pdf $g(\bullet)$ and the cdf $G(\bullet)$.

Applying Proposition 2 to our numerical example yields an average of $E\left[B_{1}\right]=$ 0.88 ships surviving the salvo, or about $15 \%$ of the starting force. This is slightly higher than the deterministic model's estimate of zero survivors. More complete information can be obtained by calculating an approximate prediction interval for the surviving force strengths using the nominal survivor mean and standard deviation, since it is the nominal distribution that is assumed to be normal. Here we interpret interval end points below zero as zero (no survivors) and above $B$ as $B$ (no losses). For example, a $90 \%$ prediction interval for $B_{1}$ is $0 \pm(1.65)(2.23)$ or from 0 to 3.67 ships. The deterministic model's point 
estimate of course gives no hint of this wide range of probable results, the equivalent of $0 \%$ to $61 \%$ of the starting force strength.

It might also be of interest to know the conditional surviving force strength, i.e. given that at least some ships survive, how many of them will there be? PROPOSITION 3: CONDITIONAL SURVIVING FORCE STRENGTH. Let $\mu_{B 1 *}, \sigma_{B 1} *^{2}$,

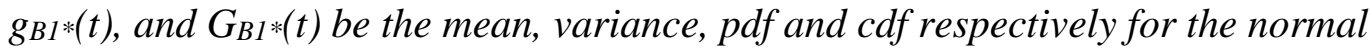
distribution that describes the nominal surviving force strength $B_{1} *$. The actual quantity of ships on side B that survive after one salvo, conditional on the knowledge that at least some survive, can be described as follows.

(a) The conditional mean surviving force strength will be

$$
E C B_{1} \equiv E\left[B_{1} \mid B_{1}>0\right]=\frac{E\left[B_{1}\right]}{1-G_{B 1^{*}}\left(0+\mu_{v} / 2\right)}
$$

(b) The conditional variance of surviving force strength will be

$$
V C B_{1} \equiv \operatorname{Var}\left[B_{1} \mid B_{1}>0\right]=\frac{\operatorname{Var}\left[B_{1}\right]+\left(E\left[B_{1}\right]\right)^{2}}{1-G_{B 1^{*}}\left(0+\mu_{v} / 2\right)}-\left(E C B_{1}\right)^{2}
$$

Using this proposition in our example shows that on those occasions where side B has at least some surviving forces, those survivors will average $E C B_{1}=1.91$ ships.

Another way to view the variety of potential outcomes is through the calculation of probabilities. We can for example look at the likelihood of various survival events after an exchange of salvos, again using the nominal cdf $G_{B 1 *}(\bullet)$ as follows.

(a) The probability of side $\mathrm{B}$ having no survivors is $\mathrm{P}\left[B_{1 *} \leq 0\right]=\left[G_{B 1} *\left(0+\mu_{v} / 2\right)\right]=54 \%$;

(b) The probability of side $\mathrm{B}$ having some but not all of their force survive is $\mathrm{P}\left[0<B_{1 *}<B\right]$ $=\left[G_{B 1} *\left(B-\mu_{v} / 2\right)-G_{B 1} *\left(0+\mu_{v} / 2\right)\right]=45 \%$;

(c) The probability of side B suffering no losses is $\mathrm{P}\left[B \leq B_{1 *}\right]=\left[1-G_{B 1 *}\left(B-\mu_{v} / 2\right)\right]=1 \%$. 
Thus whereas the deterministic model predicted $100 \%$ elimination in our example, in the stochastic model side B has a good chance of having some forces survive the exchange of fire and even a slim possibility of emerging completely unscathed.

In our numerical example the random variation affects both sides in the same way because their forces are identical; but this is not always true, especially if one force significantly outclasses the other.

PROPOSITION 4: PREFERENCES FOR VARIABILITY. The probability of side B having at least some surviving force strength $P\left[B_{1 *}>0\right]$ behaves as follows:

(a) $P\left[B_{1 *}>0\right]$ is decreasing in $\sigma_{B 1} *$ if $\mu_{B 1}>\left(0+\mu_{v} / 2\right)$, i.e., if "on average" side $B$ would have survivors anyway; but,

(a) $P\left[B_{1 *}>0\right]$ is increasing in $\sigma_{B 1} *$ if $\mu_{B 1}<\left(0+\mu_{v} / 2\right)$, i.e., if "on average" side $B$ would not have any survivors.

This proposition indicates that a superior force (i.e. one that is likely to come out ahead in a salvo exchange) will tend to prefer situations where the variation (or uncertainty) in results is relatively low. They would presumably try to fight their battles in calm weather using tried-and-true weapon systems and established tactical doctrine. Conversely, an inferior force will tend to prefer situations where the variation in results is relatively high, ideally an all-or-nothing "coin toss", and so they might try to fight their battles in rough weather using experimental technology and unorthodox tactics.

The last measure that we consider in this section is the likelihood of winning. Defining $F_{A 1} *(\bullet)$ to be the cdf of the nominal number of survivors on side A (in direct parallel to $G_{B 1 *}(\bullet)$ for side B), we can calculate the probabilities of the four possible outcomes of one exchange of salvos for our ongoing example as follows. 
(a) The probability of side A winning control is $\left[1-F_{A 1} *\left(0+\mu_{u} / 2\right)\right]\left[G_{B 1} *\left(0+\mu_{v} / 2\right)\right]=25 \%$;

(b) The probability of side B winning control is $\left[F_{A 1} *\left(0+\mu_{u} / 2\right)\right]\left[1-G_{B 1} *\left(0+\mu_{v} / 2\right)\right]=25 \%$;

(c) The probability that both sides have ships left after the salvo is

$\left[1-F_{A 1} *\left(0+\mu_{u} / 2\right)\right]\left[1-G_{B 1} *\left(0+\mu_{v} / 2\right)\right]=21 \% ;$ and,

(d) The probability of both sides being destroyed is $\left[F_{A 1} *\left(0+\mu_{u} / 2\right)\right]\left[G_{B 1}\left(0+\mu_{v} / 2\right)\right]=29 \%$.

These figures assume that each side is seeking control of the sea area (i.e. they want to end up with some friendly survivors but no enemy survivors); equivalent calculations could also be done for a force that only desires sea denial (i.e. to destroy the enemy) or that wants to simply survive. Clearly these numbers give a very different representation of the salvo's outcomes than the simpler results of the deterministic model, which gave mutual destruction (case (d) above) as the only indicated result.

\section{MODEL EXPLORATION AND INSIGHTS}

In this section we explore the behavior of the stochastic salvo model using some numerical studies. Throughout this section we set all missile success probabilities at $p_{\alpha}=$ $p_{\beta}=p_{y}=p_{z}=68 \%$, which is a figure taken from an empirical study by Schulte described by Hughes (2000, pp 275-276). Average damage is set at $\mu_{u}=\mu_{v}=1 / 3$ ships lost per missile, which corresponds to the estimated staying power of 3 hits to put out of action a ship about 500 feet long (like an Arleigh Burke DDG) according to a study by the Brookings Institution that was also described by Hughes (2000, p 161). Along with this we choose standard deviation $\sigma_{u}=\sigma_{v}=1 /(3)(2.5)$ ships so that the interval $\mu_{v} \pm 2.5 \sigma_{v}$ corresponds to a range of $0 / 3$ to $2 / 3$ ships lost per hit. The other parameters in the model are varied according to the objectives of each set of calculations described below. 
Our first observations concern the results of the stochastic model as compared to those of the deterministic model. Throughout our exploratory calculations we found that the two models tended to yield values for the average surviving force strength after a salvo that were reasonably similar. These values differed most when the two sides were closely matched (as in the illustrative example of the previous section), whereas there were no differences at all whenever either side had a large advantage.

On the other hand we did see some important differences in the other outputs of the two models. For example, Figure 1 shows the probabilities of victory for each side after one salvo as the initial force strength $B$ is gradually increased from 1 to 15 ships while the opponent is fixed at $A=6$ ships. By "victory" here we mean sea control; i.e., the enemy is eliminated while some friendly forces survive. We used identical ships for each side and set $n_{\alpha}=n_{\beta}=8$ SSMs per ship and $n_{y}=n_{z}=3$ SAMs per ship to give a relatively high level of lethality (Armstrong, 2004). We can see that the two models give similar results as long as either side has a large advantage, but not when the odds are fairly close. The deterministic model of course only allows for one possible result of any battle, and so has abrupt shifts as the force strengths change; it predicts e.g. $100 \%$ mutual destruction when each side has 6 ships, but a 100\% chance of victory by side B if it employs 7 ships. In contrast the stochastic model shows somewhat smoother transitions; under the same conditions it gives B a 19\% chance of victory when the forces are even and a $67 \%$ chance of victory when they have a one-ship advantage.

It is of course the random variation in the stochastic model that accounts for these differences, and Figure 2 illustrates this by plotting the standard deviation of the actual surviving force strengths for each side under the same conditions as in Figure 1. Neither 
side experiences much variability when the odds are extreme, as the results are nearly certain when one side is overwhelming (no losses) and the other overwhelmed (no survivors). Notice that in this high lethality example the total uncertainty actually drops when the forces are equal because it is likely that both sides will be eliminated.

Figures 3 shows a different view of the results of a salvo by graphing the probability of side A having survivors $\mathrm{P}\left[A_{1}>0\right]$, shown along the horizontal axis, together with the probability of side $\mathrm{B}$ having survivors $\mathrm{P}\left[B_{1}>0\right]$, shown along the vertical axis. For this figure we fix $A=6$ ships and then vary the opposing strength $B$ from 3 ships up to 10 ships. Both sides are assumed to have $n_{\alpha}=n_{\beta}=8$ SSMs per ship in all cases but the number of SAMs per ship is set at a different value for each curve $\left(n_{y}=n_{z}=3,4\right.$, or 5). For example, the curve running roughly across the diagonal of the diagram (marked with triangular dots and labeled SAM =4) represents the case where each ship carries 4 SAMs. The triangle located at approximately the $(61 \%, 61 \%)$ point represents the results of the particular case where $B=6$ ships; i.e. each side has a $61 \%$ chance of surviving the salvo. Movement along this curve towards the upper left occurs when we add ships to side $\mathrm{B}$, while movement along the curve to the lower right occurs when we take away ships from side B.

Thus we may think of each curve in Figure 3 as representing the decision facing side B's operational commander: given the ship types on each side, how many friendly ships should be committed to an impending battle? The implications of this decision vary according to the curve's shape: convex curves (e.g. SAM = 3) passing through the lower left quadrant indicate that additions to force strength first decrease the enemy's chance of survival and only later serve to increase the friendly survival probability. Conversely, 
concave curves (e.g. SAM = 5) passing through the upper right quadrant show that additional ships first increase friendly survival chances and only subsequently serve to decrease the enemy's chances.

Figure 4 uses the same kind of plot to examine the effect of changing the weapon mix for a fleet. We begin by again fixing $A=6$ ships, each of which fires $n_{\alpha}=8 \mathrm{SSMs}$ and $n_{y}=5$ SAMs per salvo. Each curve shown corresponds to a different number of ships on side B, either 5, 6, or 7. For each of B's ships the total firepower (SSMs plus SAMs) is kept constant at $n_{\beta}+n_{z}=13$ missiles but the mix of offensive and defensive missiles is varied over a range of SSM values from $n_{\beta}=5$ up to 13 . Thus movement along any one curve from upper right to lower left occurs as we take away one SAM and add one SSM per side B ship.

We may think of each curve in Figure 4 as characterizing the trade-off often faced by side B's naval planners: given the enemy forces we expect to encounter, with what mix of offensive and defensive abilities should our ships be equipped? The answer here depends on whether we expect to fight from a position of strength or of weakness. If our side will be superior overall (the $B=7$ case), then the concavity of the corresponding curve (marked with diamonds) indicates that a relatively balanced allocation of offensive and defensive capabilities is best, as it puts our force on the curve's "efficient frontier" in the upper left quadrant where we are highly likely to survive but almost certain to eliminate the enemy. On the other hand if we expect to be the inferior force (the $B=5$ case), then the convex curve (marked with triangles) represents our predicament. If we want a good chance of inflicting losses we can emphasize offensive power, but it will cost us our own force; the alternative is to emphasize defensive power and have a chance 
of survival, though that means the enemy is likely to escape harm as well. A balanced allocation of firepower would put our force in the lower right quadrant of the diagram: this is the worst case for an inferior force, as it would be outclassed on both offense and defense simultaneously.

Note that these decision curves are not just artifacts of our particular parameter choices, but rather arise from some fundamental relationships within the stochastic salvo model. If side B increases its total staying power or total defensive power, then clearly the likelihood of it surviving a salvo is increased; thus $\mathrm{P}\left[B_{1}>0\right]$ is increasing in side B's number of defensive missiles $n_{z}$, probability of defensive missile success $p_{z}$, and staying power $x$. Similarly, if side B increases its average offensive effectiveness then the likelihood of side A surviving a salvo is decreased; thus $\mathrm{P}\left[A_{1}>0\right]$ is decreasing in side $\mathrm{B}$ 's offensive missile capacity $n_{\beta}$, probability of offensive missile success $p_{\beta}$, and in the average damage $\mu_{u}$ suffered per hit. These effects are illustrated by the arrows in Figure 5 that show the shift in plot location (i.e. combat results) caused by an increase in each model parameter.

These figures also provide a way to understand the concept of lethality (Armstrong, 2004) in the context of the stochastic model. In the deterministic model there were three discrete levels of lethality, but Figures 3-5 instead display an infinite range of lethality levels. What we might call "strict" high lethality corresponds to an Lshaped path that runs from the lower right corner along the horizontal axis to the lower left corner, and then up the vertical axis to the upper left corner. Along this extreme path there is always at least one force that has a $100 \%$ probability of being eliminated in one salvo. Convex curves (e.g. SAM = 3) passing through the lower left quadrant approach 
this limiting extreme and indicate "relatively high" lethality, while concave curves (e.g. SAM = 5) that pass through the upper right quadrant of the diagram could be thought of as representing "moderately low" lethality conditions.

\section{CONCLUSION}

In this work we have proposed a relatively simple stochastic salvo model for naval missile combat. Starting with the existing deterministic salvo model of Hughes (1995) we substituted random variables in place of the fixed parameters for individual ships' offensive power and defensive power. In a like manner we replaced the fixed damage caused by each offensive missile by a random variable as well. We then adapted some concepts from basic stochastic inventory models to derive the mean and variance of the surviving forces on each side as well as their probabilities of winning. These measures were all provided in formulations simple enough to permit calculation using ordinary spreadsheet software.

A comparison of the stochastic model's results with those of the original deterministic model showed that both models provide similar estimates of the average number of survivors of a salvo, but because the deterministic model gives no indication of the possible variation it could be quite misleading. The stochastic model's ability to provide range estimates and the probabilities of different outcomes provides a decisionmaker with a much richer and more complete picture of the potential outcome of a salvo.

It appears that the best preparations for salvo combat depend upon whether a navy is generally inferior or superior to its opponent. Our results suggest that a superior force ought to aim for a balance of offensive and defensive capabilities, and should seek to engage in battle under conditions of greatest certainty (i.e. minimal variation or "risk"). 
Conversely, an inferior force should concentrate its resources on either offensive or defensive power (but not both) depending on its mission objectives and then seek battle under conditions of greatest uncertainty (maximum variation). Finally we also demonstrated that the concept of combat lethality analyzed by Armstrong (2004) is more complex when random variation is taken into account. In place of the three distinct categories of lethality that exist in the deterministic model we find a continuous range of lethality levels with "strict" high lethality as the limiting extreme.

One of the advantages of using deterministic rather than stochastic models is the former's simplicity. However as noted by Lucas (2003, p 9), "The nature of combat, along with fundamental mathematical principles, implies that most combat simulations should be stochastic. Although significant costs are associated with stochastic models, the resulting benefits will usually exceed the costs." We believe that for most purposes the gains from including stochastic elements in the salvo model of naval combat are well worth the costs in extra complexity. Nonetheless, our simplified stochastic salvo model itself has many limitations arising from its construction. Like the deterministic model upon which it is based, our model is highly aggregated and so implicitly assumes that incoming fire is spread evenly over all possible targets. In reality it is likely that some ships will face more fire than others, whether by accident or by design. A future study might therefore use a less aggregated model to explore the effective use of tactics and targeting information, much as was done in Lucas \& McGunnigle (2003).

Another potential limitation results from our assumption that the nominal losses and hence the nominal number of survivors of a salvo will approximately follow a normal distribution. We have argued that there are good reasons for making this assumption, but 
nevertheless it may not be appropriate in all cases. For example, it may be that in some circumstances the damage inflicted per missile might be better described by a heavily skewed distribution like the exponential. Future work might therefore investigate how this alternative could be incorporated into a salvo model's structure and whether it would have any significant impact on the model's outputs.

\section{ACKNOWLEDGEMENTS}

Thanks are due to two anonymous referees for their suggestions on improving the paper's presentation, and to Dr Roland Thomas for taking the time to discuss the mathematical properties of different probability distributions. During this research the author was funded in part by a Discovery Grant from Canada's Natural Sciences and Engineering Research Council (NSERC). 


\section{APPENDIX}

\section{The use of normal approximations.}

To see the effect of our use of normal approximations to the underlying binomial distributions, we calculated the mean number of actual hits using our model (i.e. Equation 6 but with $\sigma_{v}^{2}=0$, i.e. deterministic damage) for a number of situations. We then compared these results to direct calculations using the difference of the two underlying binomial distributions. We discovered that both methods gave similar results, even for small battles; Figure 6 shows examples of our findings. Each row corresponds to a different missile match-up, and each column corresponds to a different probability of missile success (using the same probability for both offensive and defensive fire). For example, when 3 SSMs were fired against 6 SAMs, and each missile had an $80 \%$ probability of success, then the model's normal calculation gave a mean number of hits (0.0070) that was $31 \%$ lower than the binomial calculations (0.0102). The large $\%$ value occurs because in situations dominated by defensive fire a hit only occurs when both the offense gets lucky and the defense is unlucky, i.e. we are doing calculations using the tails of the respective distributions, where the fit between them is worst. Fortunately this large relative difference is largely irrelevant for users of the model because the absolute difference between the two calculations for this case (and for all the other cases shown in the figure) is less than 0.01 hits.

Table 2. Relative difference in mean actual number of hits.

\begin{tabular}{|lrrrrr|}
\hline & $p=0.80$ & $p=0.68$ & $p=0.50$ & $p=0.32$ & $p=0.20$ \\
\hline 6 SSM v 3 SAM & $-0.4 \%$ & $-0.6 \%$ & $-0.7 \%$ & $-0.8 \%$ & $-0.8 \%$ \\
\hline 6 SSM v 6 SAM & $-1.7 \%$ & $-2.0 \%$ & $-2.1 \%$ & $-2.0 \%$ & $-1.7 \%$ \\
\hline 3 SSM v 6 SAM & $-31.0 \%$ & $-7.9 \%$ & $-1.9 \%$ & $-0.4 \%$ & $1.7 \%$ \\
\hline
\end{tabular}




\section{Transforming integrals into convenient forms.}

In the proofs that follow we often need to transform integrals involving a normal pdf, so these are given below for reference. In each case, $F(t)$ and $f(t)$ are the cdf and pdf, respectively, of a normal distribution with mean $\mu$ and variance $\sigma^{2}$.

Integral of $f(t)$.

$$
\int_{T 1}^{T 2} f(t) d t=\left[F\left(T_{2}\right)-F\left(T_{1}\right)\right]
$$

Proof: Follows from the definition of the cdf $F(t)$.

Integral of $t f(t)$.

$$
\int_{T 1}^{T 2} t f(t) d t=\mu\left[F\left(T_{2}\right)-F\left(T_{1}\right)\right]-\sigma^{2}\left[f\left(T_{2}\right)-f\left(T_{1}\right)\right]
$$

Proof: A known result in inventory theory, see e.g. Chopra \& Meindl (2004, p 340).

Integral of $t^{2} f(t)$.

$$
\int_{T 1}^{T 2} t^{2} f(t) d t=\left(\mu^{2}+\sigma^{2}\right)\left[F\left(T_{2}\right)-F\left(T_{1}\right)\right]-\sigma^{2}\left[\left\langle T_{2}+\mu\right\rangle f\left(T_{2}\right)-\left\langle T_{1}+\mu\right\rangle f\left(T_{1}\right)\right]
$$

Proof: Write out the expression in full and then divide it into three sections.

$$
\begin{gathered}
\int_{T 1}^{T 2} t^{2} f(t) d t=\frac{1}{\sqrt{2 \pi \sigma^{2}}} \int_{T 1}^{T 2} t^{2} e^{-\frac{1}{2}\left(\frac{t-\mu}{\sigma}\right)^{2}} d t \\
=\frac{1}{\sqrt{2 \pi \sigma^{2}}} \int_{T 1}^{T 2}\left[\left(\mu^{2}+\sigma^{2}\right)+\left(t \mu-\mu^{2}\right)+\left(t^{2}-t \mu-\sigma^{2}\right)\right] e^{-\frac{1}{2}\left(\frac{t-\mu}{\sigma}\right)^{2}} d t
\end{gathered}
$$

In the first section apply A1.

$$
\left(\mu^{2}+\sigma^{2}\right) \frac{1}{\sqrt{2 \pi \sigma^{2}}} \int_{T 1}^{T 2} e^{-\frac{1}{2}\left(\frac{t-\mu}{\sigma}\right)^{2}} d t=\left(\mu^{2}+\sigma^{2}\right)\left[F\left(T_{2}\right)-F\left(T_{1}\right)\right] .
$$

For the second section use A2 and A1. 


$$
\begin{gathered}
\frac{1}{\sqrt{2 \pi \sigma^{2}}} \int_{T 1}^{T 2}\left(t \mu-\mu^{2}\right) e^{-\frac{1}{2}\left(\frac{t-\mu}{\sigma}\right)^{2}} d t=\frac{\mu}{\sqrt{2 \pi \sigma^{2}}} \int_{T 1}^{T 2} t e^{-\frac{1}{2}\left(\frac{t-\mu}{\sigma}\right)^{2}} d t-\frac{\mu^{2}}{\sqrt{2 \pi \sigma^{2}}} \int_{T 1}^{T 2} e^{-\frac{1}{2}\left(\frac{t-\mu}{\sigma}\right)^{2}} d t \\
=-\sigma^{2}\left[\mu f\left(T_{2}\right)-\mu f\left(T_{1}\right)\right]
\end{gathered}
$$

The third section is resolved as follows.

$$
\begin{gathered}
\frac{1}{\sqrt{2 \pi \sigma^{2}}} \int_{T 1}^{T 2}\left(t^{2}-t \mu-\sigma^{2}\right) e^{-\frac{1}{2}\left(\frac{t-\mu}{\sigma}\right)^{2}} d t=\frac{\sigma^{2}}{\sqrt{2 \pi \sigma^{2}}} \int_{T 1}^{T 2}\left(\frac{t(t-\mu)}{\sigma^{2}}-1\right) e^{-\frac{1}{2}\left(\frac{t-\mu}{\sigma}\right)^{2}} d t \\
=\frac{\sigma^{2}}{\sqrt{2 \pi \sigma^{2}}}\left[-T_{2} e^{-\frac{1}{2}\left(\frac{t-\mu}{\sigma}\right)^{2}}+T_{1} e^{-\frac{1}{2}\left(\frac{t-\mu}{\sigma}\right)^{2}}\right]=-\sigma^{2}\left[T_{2} f\left(T_{2}\right)-T_{1} f\left(T_{1}\right)\right]
\end{gathered}
$$

Combine the three sections and re-group the terms to yield the desired result.

\section{Proof for Lemma 1: Variance of a random sum.}

The proof is presented only for the case where $L$ is a continuous random variable. For part (a) begin by noting that

$$
E\left[M^{2} \mid L=l\right]=\operatorname{Var}[M \mid L=l]+(E[M \mid L=l])^{2}=|l| \sigma_{K}{ }^{2}+l^{2} \mu_{K}{ }^{2} .
$$

Set up the variance definition and work with its components.

$$
\begin{gathered}
\operatorname{Var}[M]=E\left[M^{2}\right]-E[M]^{2}=\int_{-\infty}^{\infty} E\left[M^{2} \mid L=l\right] g(l) d l-\left(\mu_{L} \mu_{K}\right)^{2} \\
=\int_{-\infty}^{\infty}\left(|l| \sigma_{K}{ }^{2}+l^{2} \mu_{K}^{2}\right) g(l) d l-\mu_{L}{ }^{2} \mu_{K}{ }^{2} \\
=\sigma_{K}{ }^{2}\left\{\int_{0}^{\infty} l g(l) d l+\int_{-\infty}^{0}-\lg (l) d y\right\}+\mu_{K}{ }^{2} E\left[L^{2}\right]-\mu_{L}{ }^{2} \mu_{K}{ }^{2} \\
=\sigma_{K}{ }^{2}\left\{E[L]-2 \int_{-\infty}^{0} \lg (l) d l\right\}+\mu_{K}{ }^{2}\left(\mu_{L}{ }^{2}+\sigma_{L}{ }^{2}\right)-\mu_{L}{ }^{2} \mu_{K}{ }^{2}
\end{gathered}
$$

Part (b) is obtained using A2. 


$$
-2 \sigma_{K}^{2} \int_{-\infty}^{0} \lg (l) d l=-2 \sigma_{K}^{2}\left\{\mu_{L}[G(0)-0]-\sigma_{L}^{2}[g(0)-0]\right\}
$$

\section{Proof for Proposition 1: Nominal surviving force strength.}

Part (a) for the mean is straightforward. For part (b), note that the variance of the survivors is equal to the variance of the losses, which is obtained via Lemma 1.

$$
\begin{gathered}
\operatorname{Var}\left[B_{1}{ }^{*}\right]=\mathrm{E}\left[N e t_{A B}\right] \sigma_{v}{ }^{2}+\operatorname{Var}\left[N e t_{A B}\right] \mu_{v}{ }^{2}-2 \sigma_{v}{ }^{2} \int_{-\infty}^{0} t g_{N e t A B}(t) d t \\
=\left[A \mu_{\alpha}-B \mu_{z}\right] \sigma_{v}{ }^{2}+\left[A \sigma_{\alpha}{ }^{2}-B \sigma_{z}{ }^{2}\right] \mu_{v}{ }^{2}-2 \sigma_{v}{ }^{2} G_{N e t A B}(0)\left[A \mu_{\alpha}-B \mu_{z}\right]+2{\sigma_{v}}^{2} g_{N e t A B}(0)\left[A \sigma_{\alpha}{ }^{2}-B \sigma_{z}{ }^{2}\right]
\end{gathered}
$$

\section{Proof for Proposition 2: Actual surviving force strength.}

Part (a) needs $\mathrm{E}\left[\min \left\{B, \max \left\{0, B_{1}\right\}\right\}\right]$.

$$
E\left[B_{1}\right]=0+\int_{0+\mu_{v} / 2}^{B-\mu_{v} / 2} t g_{B 1^{*}}(t) d t+\int_{B-\mu_{v} / 2}^{\infty} B g_{B 1^{*}}(t) d t=\int_{0+\mu_{v} / 2}^{B-\mu_{v} / 2} t g_{B 1^{*}} d t+B\left(1-G_{B 1^{*}}\left(B-\mu_{v} / 2\right)\right)
$$

Apply A2 to the integral.

$$
\begin{gathered}
=\mu_{B 1^{*}}\left[G_{B 1^{*}}\left(B-\mu_{v} / 2\right)-G_{B 1^{*}}\left(0+\mu_{v} / 2\right)\right]-\sigma_{B 1^{*}}^{2}\left[g_{B 1^{*}}\left(B-\mu_{v} / 2\right)-g_{B 1^{*}}\left(0+\mu_{v} / 2\right)\right] \\
+B\left[1-G_{B 1^{*}}\left(B-\mu_{v} / 2\right)\right]
\end{gathered}
$$

Part (b) obtains $\operatorname{Var}\left[\min \left\{B, \max \left\{0, B_{1}\right\}\right\}\right]$ in a similar manner but using A3.

\section{Proof for Proposition 3: Conditional surviving force strength.}

In part (a) the mean is determined using the conditional distribution.

$$
\begin{aligned}
E & {\left[B_{1} \mid B_{1}>0\right]=E\left[B_{1} \mid B_{1}^{*}>\left(0+\mu_{v} / 2\right)\right] } \\
& =\int_{0+\mu_{v} / 2}^{B-\mu_{v} / 2} t \frac{g_{B 1^{*}}(t)}{1-G_{B 1^{*}}\left(0+\mu_{v} / 2\right)} d t+B \frac{1-G_{B 1^{*}}\left(B-\mu_{v} / 2\right)}{1-G_{B 1^{*}}\left(0+\mu_{v} / 2\right)}
\end{aligned}
$$

The conditional variance for part (b) is derived in a similar manner.

$$
\operatorname{Var}\left[B_{1} \mid B_{1}>0\right]=\int_{0+\mu_{v} / 2}^{B-\mu_{v} / 2} t^{2} \frac{g_{B 1^{*}}(t)}{1-G_{B 1^{*}}\left(0+\mu_{v} / 2\right)} d t+B^{2} \frac{1-G_{B 1^{*}}\left(B-\mu_{v} / 2\right)}{1-G_{B 1^{*}}\left(0+\mu_{v} / 2\right)}-\left(E C B_{1}\right)^{2}
$$




$$
\begin{gathered}
=\frac{1}{1-G_{B 1^{*}}\left(0+\mu_{v} / 2\right)}\left\{\int_{0+\mu_{v} / 2}^{B-\mu_{v} / 2} t^{2} g_{B 1^{*}}(t) d t+B^{2}\left[1-G_{B 1}\left(B-\mu_{v} / 2\right)\right]\right\}-\left(E C B_{1}\right)^{2} \\
=\frac{E\left(B_{1}{ }^{2}\right)}{1-G_{B 1^{*}}\left(0+\mu_{v} / 2\right)}-\left(E C B_{1}\right)^{2}
\end{gathered}
$$

\section{Proof for Proposition 4: Preferences for Variance.}

The probability of side B having survivors is

$$
P\left[B_{1}>0\right]=P\left[B_{1}^{*}>\left(0+\mu_{v} / 2\right)\right]=\int_{0+\mu_{v} / 2}^{\infty} \frac{1}{\sqrt{2 \pi \sigma_{B 1^{*}}^{2}}} e^{\left.-\left(t-\mu_{B 1^{*}}\right)^{2} /\left(2 \sigma_{B 1^{*}}\right)^{2}\right)} d t .
$$

Take the derivative with respect to the standard deviation.

$$
\frac{\partial}{\partial \sigma_{B 1^{*}}}\left\{P\left[B_{1}>0\right]\right\}=\frac{-1}{\sigma_{B 1^{*}}} \int_{0+\mu_{v} / 2}^{\infty} g_{B 1^{*}}(t) d t+\frac{1}{\sigma_{B 1^{*}}^{2}} \int_{0+\mu_{v} / 2}^{\infty}\left(\mu_{B 1^{*}}^{2}-2 t \mu_{B 1^{*}}+t^{2}\right) g_{B 1^{*}}(t) d t
$$

Transform the 1st integral with A1, and likewise the 2nd integral with A1, A2 and A3.

$$
\begin{aligned}
= & \frac{-1}{\sigma_{B 1^{*}}}\left[1-G_{B 1^{*}}\left(0+\mu_{v} / 2\right)\right]+\frac{1}{\sigma_{B 1^{*}}{ }^{2}} \mu_{B 1^{*}}{ }^{2}\left[1-G_{B 1^{*}}\left(0+\mu_{v} / 2\right)\right] \\
& -\frac{2 \mu_{B 1^{*}}}{\sigma_{B 1^{*}}{ }^{2}}\left\{\mu_{B 1^{*}}\left[1-G_{B 1^{*}}\left(0+\mu_{v} / 2\right)\right]-\sigma_{B 1^{*}}{ }^{2}\left[0-g_{B 1^{*}}\left(0+\mu_{v} / 2\right)\right]\right\} \\
& +\frac{1}{\sigma_{B 1^{*}}{ }^{2}}\left\{\left(\mu_{B 1^{*}}{ }^{2}+\sigma_{B 1^{*}}{ }^{2}\right)\left[1-G_{B 1^{*}}\left(0+\mu_{v} / 2\right)\right]-\sigma_{B 1^{*}}{ }^{2}\left[0-\left(0+\mu_{v} / 2+\mu_{B 1^{*}}\right) g_{B 1^{*}}\left(0+\mu_{v} / 2\right)\right]\right\}
\end{aligned}
$$

Cancel terms and simplify to get the following.

$$
=\frac{1}{\sigma_{B 1^{*}}}\left[\mu_{B 1^{*}}-\left(0+\mu_{v} / 2\right)\right] g_{B 1^{*}}\left(0+\mu_{v} / 2\right)
$$

If $\mu_{B 1 *}>\left(0+\mu_{v} / 2\right)$ then this expression will be negative, indicating that $\mathrm{P}\left[B_{1}>0\right]$ is decreasing in $\sigma_{B 1} *$. Conversely, if $\mu_{B 1}<\left(0+\mu_{v} / 2\right)$ then the expression will be negative, indicating that $\mathrm{P}\left[B_{1}>0\right]$ is increasing in $\sigma_{B 1 *}$. 


\section{REFERENCES}

Ancker CJ, 1995, A proposed foundation for a theory of combat, Naval Research Logistics 42, 311-343.

Armstrong MJ, 2004, Effects of Lethality in Naval Combat Models, Naval Research Logistics 51, 28-43.

Bagchi U, Hayya JC, Chu CH, 1986, The effect of lead-time variability: the case of independent demand, Journal of Operations Management 6, 159-177.

Chopra S, Meindl P, 2004, Supply Chain Management, $2^{\text {nd }}$ ed, Prentice Hall.

Eddy P, Linklater M, Gillman G, 1982, War in the Falklands, Fitzhenry \& Whiteside. Hadley G, Whitin TM, 1963, Analysis of Inventory Systems, Prentice Hall. Hughes WP, 1995, A salvo model of warships in missile combat used to evaluate their staying power, Naval Research Logistics 42, 267-289.

Hughes WP, 2000, Fleet tactics and coastal combat, Naval Institute Press, Annapolis. Johns MD, Pilnick SE, Hughes WP, 2001, Heterogeneous salvo model for the navy after next, technical report NPS-IJWA-01-010, Naval Postgraduate School, Monterey.

Johnson NL, Kotz S, 1969, Discrete Distributions, Houghton Mifflin.

Kent G, 2002, Looking back: four decades of analysis, Operations Research 50, 122-124.

Kress M, Talmor I, 1999, A new look at the 3:1 rule of combat through Markov stochastic Lanchester models, Journal of the Operational Research Society 50, 733-744. Lucas TW, 2000, The stochastic versus deterministic argument for combat simulations: tales of when the average won't do, Military Operations Research 5, \#3, 9-28. 
Lucas TW, McGunnigle JE, 2003, When is model complexity too much? Illustrating the benefits of simple models with Hughes' salvo equations, Naval Research Logistics 50, 197-217.

Nahmias S, 1993, Production and Operations Analysis, 2nd ed, Irwin Inc.

Ross SM, 1993, Probability Models, 5th ed, Harcourt Brace \& Company.

Taylor JG, 1983, Lanchester Models of Warfare, Operations Research Society of America. 
Table 1: Summary of Notation.

The first symbol in each pair refers to side A, the second to side B.

\begin{tabular}{|ll|}
\hline Symbol & Description \\
\hline$A, B$ & Beginning force strength \\
$\alpha_{i}, \beta_{i}$ & Offensive power per unit \\
$w, x$ & Staying power per unit \\
$y_{j}, z_{j}$ & Defensive power per unit \\
$u_{k}, v_{k}$ & Losses per hit \\
$n_{\alpha}, n_{\beta}$ & Maximum \# of offensive missiles per ship per salvo \\
$p_{\alpha}, p_{\beta}$ & P[an offensive missile is successfully well-targeted] \\
$O f f_{A}, O f f_{B}$ & Total \# of well-targeted offensive missiles per salvo \\
$n_{y}, n_{z}$ & Maximum \# of defensive missiles per ship per salvo \\
$p_{y}, p_{z}$ & P[a defensive missile successfully intercepts] \\
$D e f_{A}, D e f_{B}$ & Total \# of accurate defensive missiles per salvo \\
$N e t_{B A}, N e t_{A B}$ & Total nominal \# of non-intercepted offensive missiles \\
$F_{N e t B A}, G_{N e t A B}$ & cdf of nominal \# of non-intercepted offensive missiles \\
$f_{N e t B A}, g_{N e t A B}$ & pdf of nominal \# of non-intercepted offensive missiles \\
$A_{1} *, B_{1} *$ & Nominal surviving force strength after one salvo \\
$F_{B 1^{*}, G_{B 1}}$ & cdf of nominal surviving force strength \\
$f_{B 1^{*}}, g_{B 1^{*}}$ & pdf of nominal surviving force strength \\
$A_{1}, B_{1}$ & Actual surviving force strength after one salvo \\
\hline
\end{tabular}

Figure 5. Effects of an increasing parameter on survival probabilities.

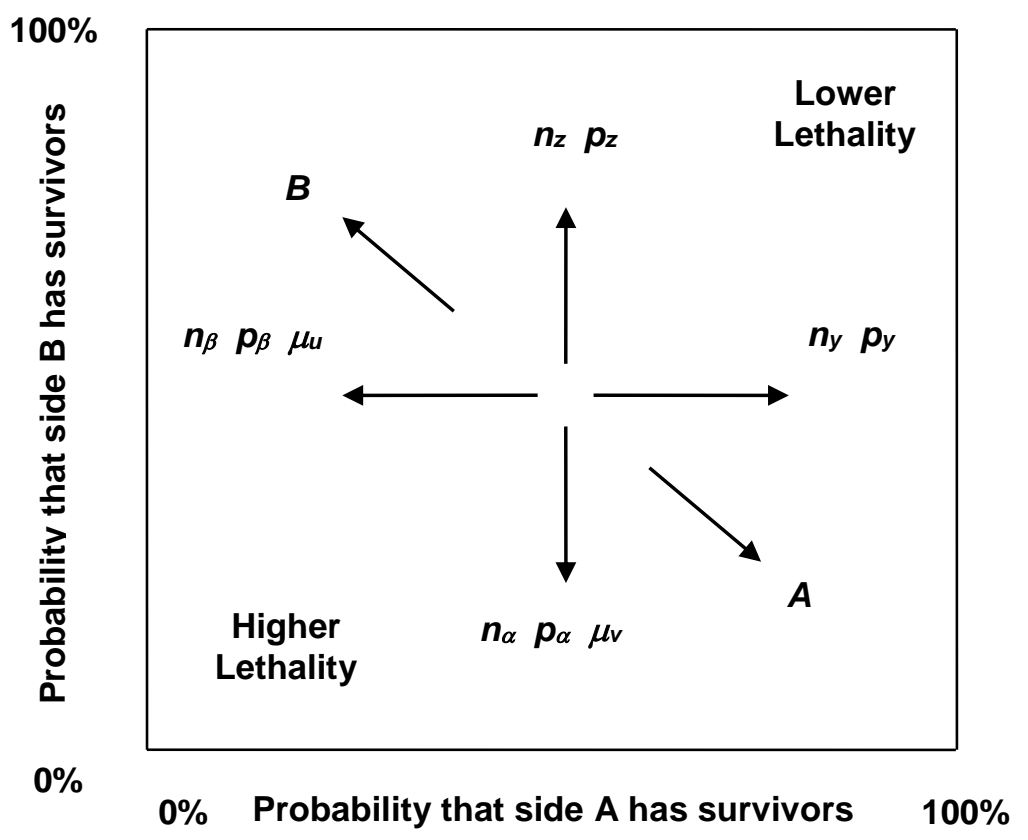


Figure 1. Probability of victory as force strength $B$ is varied.

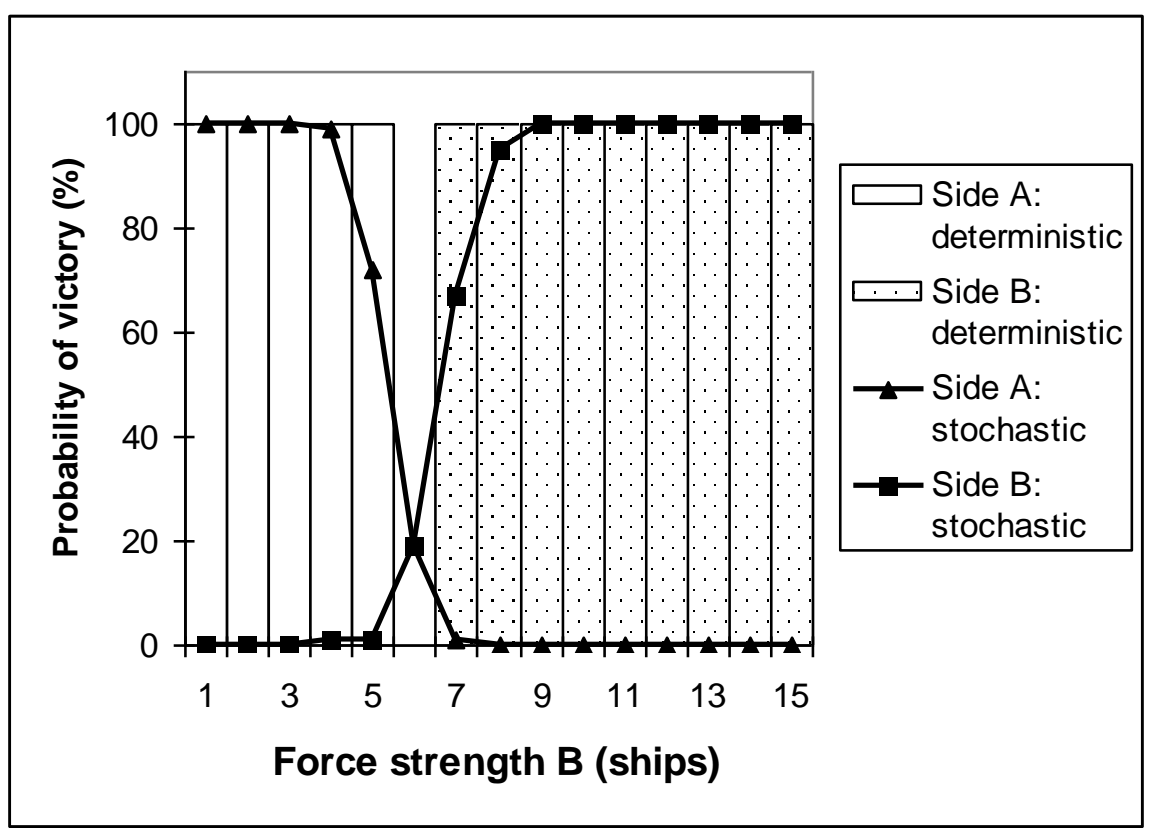

Figure 2. Standard deviation of survivors as force strength B is varied.

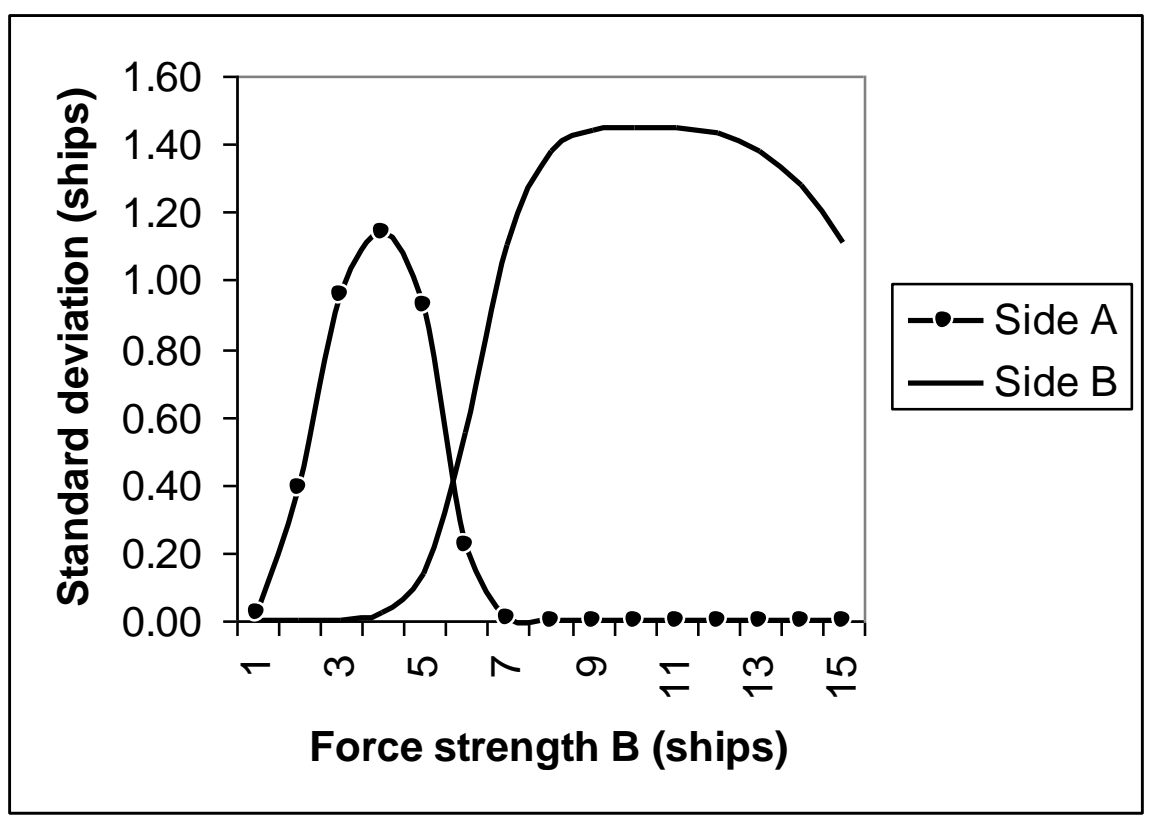


Figure 3. Probabilities of survival as force strength $B$ is varied.

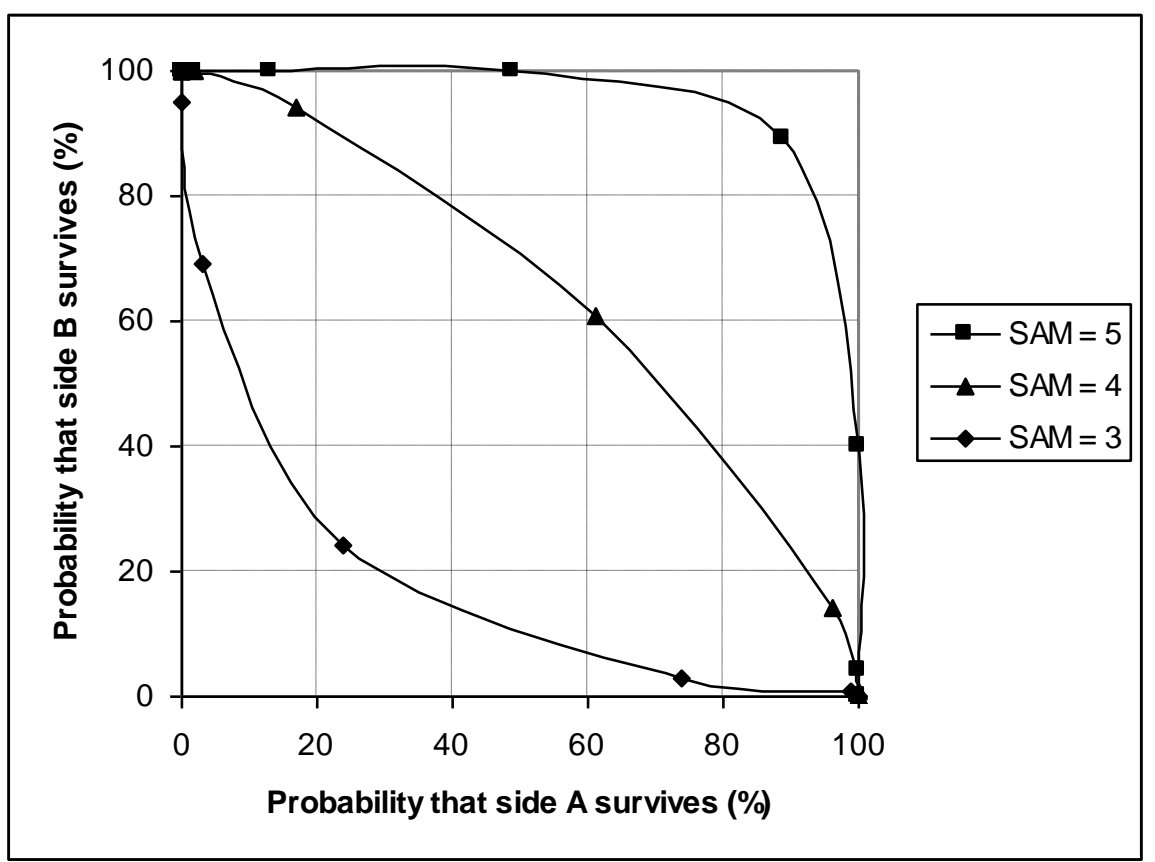

Figure 4. Probabilities of survival as missile load for $B$ is varied.

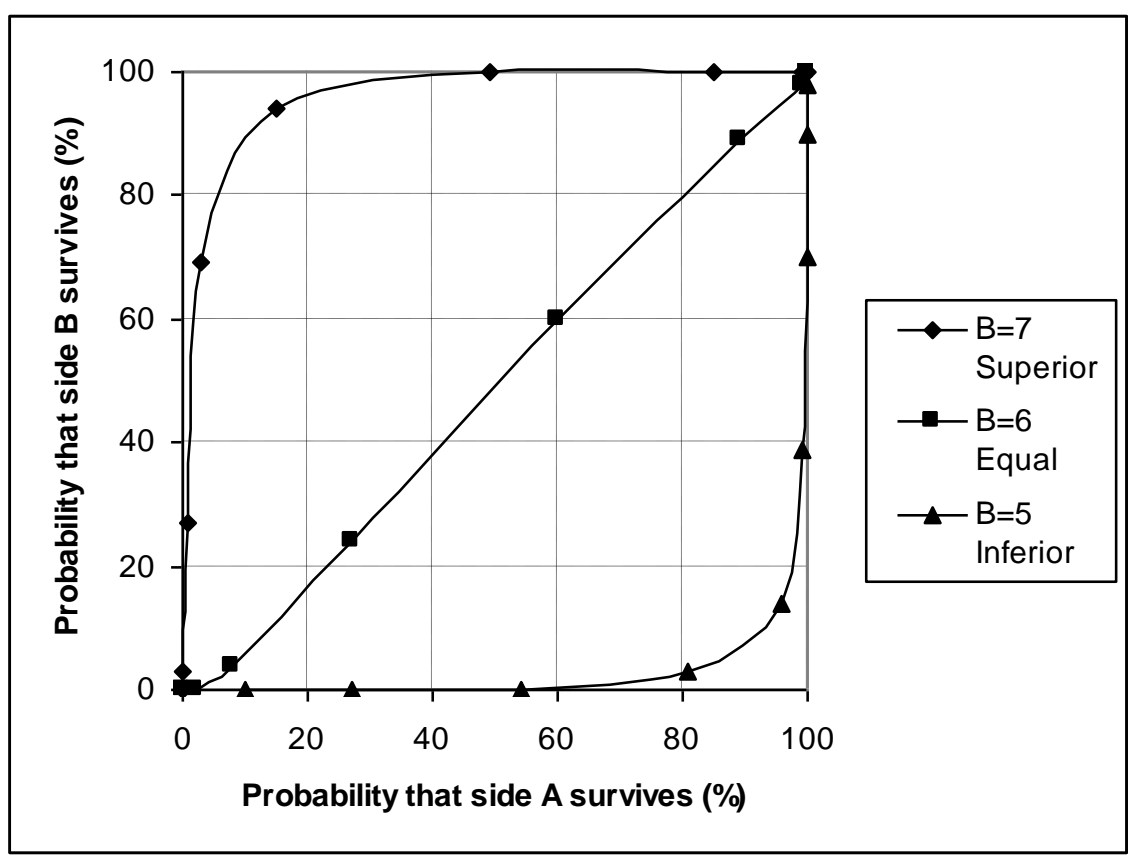

